\title{
Excitation Mechanisms of the Oxygen 5577 Emission in the Upper Atmosphere'
}

\author{
E. Tandberg-Hanssen and F. E. Roach
}

(June 18, 1959)

\begin{abstract}
Possible excitation mechanisms for the green 5577 emission are considered in the light of recent data on the dynamics of the upper atmosphere. Photochemical reactions as affected by mass motions as well as excitation directly due to the mass motions are analyzed. It is concluded that either or both mechanisms could probably account for the observed emission.
\end{abstract}

\section{Introduction}

It is now well established that the bulk of the $\lambda 5577$ airglow is generated in a fairly thin layer of the atmosphere at a height of about $100 \mathrm{~km}$ (Heppner, Stolarik, and Meredith [1];2 Roach, Megill, Rees, and Marovich [2]; and Tousey [3]). Furthermore, it is known that the airglow ${ }^{3}$ is not emitted in a uniform static layer, but that the emission is concentrated in cells that move relative to the earth's surface (Roach, Tandberg-Hanssen, and Megill $[4,5]$.

A general theory of the 5577 night airglow which includes both the microscopic (excitation) mechanism and the macroscopic (dynamical cells) features is required, and in this paper two different possibilities are examined.

The principal observational facts any theory will have to explain are summarized:

(1) The average night intensity of the 5577 emission corresponds to approximately $2 \times 10^{8}$ transitions $/ \mathrm{cm}^{2}$ column sec. For a night of roughly $10 \mathrm{hr}$ duration, $2 \times 10^{8} \times 3.6 \times 10^{4}=7 \times 10^{12}$ transitions $/ \mathrm{cm}^{2}$ column are consequently needed.

(2) The airglow intensity varies during one night sometimes by as much as a factor 5 , and the maximum comes generally not in the beginning of the night (Roach, Tandberg-Hanssen, and Megill [4]).

(3) The emission is not uniform over the sky, but appears in "cells" having horizontal motions of the order of $100 \mathrm{~m} / \mathrm{sec}$.

The energy necessary to excite the ${ }^{1} \mathrm{~S}$ level of the oxygen atom (from which the atom may jump to the ${ }^{1} \mathrm{D}$ state and emit the green line $)$ is $4.2 \mathrm{ev}\left(32,000^{\circ}\right.$ $\mathrm{K})$. The temperature in the $100-\mathrm{km}$ region is less than $300^{\circ} \mathrm{K}$ which corresponds to $0.04 \mathrm{ev}$, so it is impossible that any significant excitation can be brought about by the thermal motions of the par-

1 High Altitude Observatory, University of Colorado and National Bureau of Standards, Boulder Laboratories.

2 Figures in brackets indicate the literature references at the end of this paper. 3 The following is restricted uniquely to the green emission, $\lambda 5577$. ticles. A static theory must therefore draw on exothermic photochemical reactions which are considered in section 2. Similarly, a dynamic theory must invoke environmental effects whereby energy is given perferentially to selected particles, as discussed in section 4 .

\section{Photochemical Reactions}

Above $100 \mathrm{~km}$ most atmospheric oxygen is in the atomic form, and according to Seaton [6], the most important recombination mechanism will be of the 3-body type, as proposed by Chapman [7]. This conclusion is adopted here and the Chapman reaction is considered as the most favorable photochemical excitation mechanism for the green oxygen line. The reaction may be written

$$
0+0+0 \rightarrow 0\left({ }^{1} \mathrm{~S}\right)+0_{2} .
$$

The reaction coefficient $k$ for reaction (1) may probably be written (Bates [8])

$$
k=5 \times 10^{-34} T^{\frac{1}{2}} \mathrm{~cm}^{6} / \mathrm{sec} .
$$

There is, however, no direct measurement of $k$ and the dependence $T^{\frac{1}{2}}$ is purely conventional. The yield of oxygen atoms excited to the ${ }^{1} \mathrm{~S}$ state is given by

$$
Y\left({ }^{1} \mathrm{~S}\right)=p k[n(0)]^{3} \mathrm{~cm}^{-3} \mathrm{sec}^{-1}
$$

where $p$ is an "efficiency factor," giving the fraction of 3-body collisions (1) that lead to excitation of the ${ }^{1} \mathrm{~S}$ state, and $n(0)$ is the number density of atomic oxygen.

Since the distribution of excited atoms and molecules over the different energy levels is not known, the value of $p$ is not known. In these numerical calculations, the simplifying assumption is made that $p=1 / n$ where $n$ is the number of different types of reactions (1). Taking $p=0.2$ may be a large efficiency factor, but it is at present probably the best. 
Because of the 3-body collision, the airglow emission will be critically height dependent if the distribution of atomic oxygen is at all sharply peaked at any height. If $\tau_{c}$ is the lifetime of the ${ }^{1} \mathrm{~S}$ level for collisional deactivation and $\tau_{r}$ is the radiative lifetime, then the number of photons emitted at any level by metastable oxygen atoms by (1) is proportional (Bates and Massey [9]) to

$$
\frac{[n(0)]^{3} \tau_{c}}{\tau_{c}+\tau_{r}}
$$

Detailed calculations show (Nicolet [10]) that $n(0)$ is peaked near $100 \mathrm{~km}$. According to (2) one should hence expect a pronounced maximum in the green emission near $100 \mathrm{~km}$, and this is in agreement with observations. The number of atoms formed per square centimeter column per second in the ${ }^{1} \mathrm{~S}$ state is then given by

$$
N\left({ }^{1} \mathbf{S}\right)=\int_{0}^{\infty} Y\left({ }^{1} \mathbf{S}\right) d z=p \int_{0}^{\infty} k[n(0)]^{3} d z .
$$

To take into account the actual distribution of $n(0)$ with height may be quite complicated. On the other hand, it is known that it is sharply peaked at $z=100$ $\mathrm{km}$, so most of the contribution from the integral in eq (5) comes from that height. The distribution can hence probably be well approximated with a cosine function or an exponential. An exponential model with a peak value of $n(0)$, taken from Nicolet's [10] work, is used here. Putting:

$$
n(0)=n_{\max }(0) e^{-z / H}
$$

where $H$ is the scale height at $100 \mathrm{~km}$, we find

$$
\begin{gathered}
N\left({ }^{1} \mathrm{~S}\right)=p k\left[n_{\max }(0)\right]^{3} \int_{0}^{\infty} e^{-3 z / H} d z \\
=1 / 3 K\left[n_{\max }(0)\right]^{3} H=1 / 3 Y_{0}\left[\left({ }^{1} \mathrm{~S}\right) H ; K=p k .\right.
\end{gathered}
$$

Now, according to Garstang [11], the radiative lifetime $\tau_{r}$ for the ${ }^{1} \mathrm{~S}$ state is $0.74 \mathrm{sec}$, and it is conceivable that collisional deactivation could take place before the $N\left({ }^{1} \mathrm{~S}\right)$ atoms jumped to a lower state and emitted the green line. Calling the probability of deactivation $d_{3}$, the number of transitions ${ }^{1} \mathrm{~S} \rightarrow{ }^{1} \mathrm{D}$ is given by

$$
\begin{aligned}
& N(5577)=1 / 3 Y_{0}\left({ }^{1} \mathrm{~S}\right) H\left(1-d_{3}\right) \\
& \text { transitions } / \mathrm{cm}^{2} \text { column sec. }
\end{aligned}
$$

The different quantities entering eq (6) are discussed in order to see what numerical values for the green intensity are to be expected from the Chapman reaction.

(a) Reaction coefficient, $k$.

There is no evidence of the effectiveness of the Chapman reaction (see Bates [8]) so the best is to suppose that it is of the same order of magnitude as for other reactions of this type.

For $T=200^{\circ} \mathrm{K}$, eq $(2)$ gives $k \approx 7 \times 10^{-33}$

(b) Density of atomic oxygen, $n(0)$.

The best estimates (see Nicolet [10]) indicate that $n_{\max }(0)=1.5 \times 10^{12} \mathrm{~cm}^{-3}$ between 90 and $105 \mathrm{~km}$, and the density drops off sharply below and above this region.

\section{(c) Scale height, $H$.}

The hydrostatic scale height $H=k T / m g$ at this height in the atmosphere is $H=6.5 \mathrm{~km}$ (U.S. Extension to the ICAO Standard Atmosphere [12]).

\section{(d) Probability of deactivation, $d_{3}$.}

The problem of deactivation of the ${ }^{1} \mathrm{~S}$ level has mainly been considered in connection with the intensity ratio of the red to the green emission under auroral conditions. In view of the comparatively short radiative lifetime of the ${ }^{1} \mathrm{~S}$ level, it has generally been assumed that collisional deactivation of this level may be neglected, i.e., $d_{3}=0$ (see Seaton [13]).

Whereas deactivation by electron collisions may be of importance for bright auroral displays (Seaton [13]), the electron concentrations required seem to be too high for the process to be of importance under ordinary airglow conditions. The rate of recombination $\left(0+0 \rightarrow 0_{2}\right)$ is so slow that there is a negligible decrease in the atomic concentration during the night (Bates and Massey [9]).

In view of the uncertainties in the data, a deactivation of the ${ }^{1} \mathrm{~S}$ level cannot be ruled out. However, it is felt here that there is no obvious process by which the ${ }^{1} \mathrm{~S}$ level will be collisionally depopulated. The factor $\left(1-d_{3}\right)$ is therefore probably close to unity, and this value will be adopted here.

Numerical values for the quantities in (6) will now be put in to see what intensities are to be expected for the green airglow according to the 3-body collision theory. Figure 1 shows the intensities $Q$ in ray-

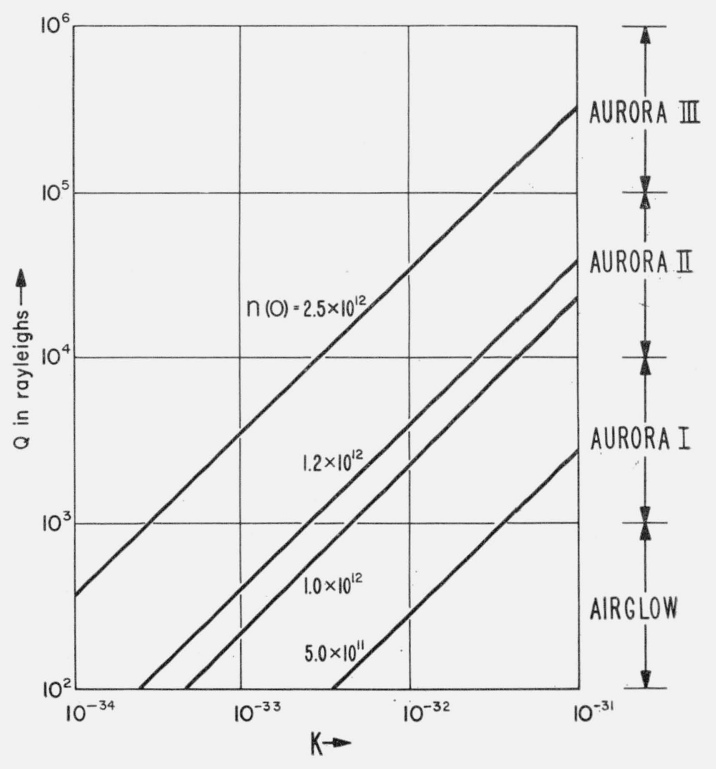

FIgure 1. Predicted intensity of [OI] 5577 from eq $(5 b)$. 
leighs ${ }^{4}$ as a function of $K$ for different values of $n(0)$, boxing in the probable range of these parameters. It is seen that already the minimum probable values give a very bright airglow. Either the yield of excited oxygen atoms, (3), has been overestimated, or the importance of collisional deactivation has been underestimated. If neither is the case, it is safe to state that within the accuracy of these data, the Chapman reaction can not only explain the observed airglow intensities, but it actually predicts a faint aurora (1100 rayleighs). However, in addition to explaining the intensity level, a successful theory also has to account for the time variations of the intensity. On a purely static picture, it is not possible to understand why the intensity maximum. does not come in the beginning of the night. Since high wind speeds of the emitting cells have been deduced the dynamics of the airglow emission into the photochemical theory will now be introduced to explore the possibility of nocturnal time variations as observed.

\section{Meterological Approach}

As long as airglow is considered as a steady, static, more or less homogeneous emission from a layer at about $100 \mathrm{~km}$, it is natural to try to explain it in terms of a pure photochemical process solely governed by the kinetic properties of the atmosphere. With the introduction of a dynamical picture in which the airglow emission is concentrated in cells that move relative to the earth's surface, one is led to ask whether the very dynamics of the emissions have anything to do with the excitation of the phenomenon.

At least two avenues lead from here. First, one may ask whether the dynamics of the upper atmosphere can modify the emissions caused by the photochemical reactions to a sufficient degree to account for the observed morphology of the airglow. This may be termed the meteorological approach. Second, one may go a step further and inquire into the possibility of using the dynamics also for the excitation of the oxygen atoms. In this case, the energy of the environmental effects is used as in the next section.

In light of the increased interest of recent years in the study of solar-weather relationships and in the possibility of a coupling between the troposphere and high atmosphere (including the lower part of the ionosphere), it seems appropriate to consider in some detail the meteorological approach to the airglow problem.

The existence of cells in the airglow emission is probable, and from a meteorological point of view, they may be considered as "clouds." The very concept of clouds is synonomous with instability, and the first question is whether one would expect instability in the high atmosphere similar to that in the troposphere. Fleagle [14] has recently shown that disturbances tend to grow rapidly in the mesodecline,

${ }^{4}$ If the brightness, $B$, is measured in units of $10^{6}$ quanta $/ \mathrm{cm}^{2}$ second steradian, then the brightness in rayleighs $(R)$ is $4 \pi B$. at least in polar regions. Also, it should be remembered that the outstanding feature of the troposphere in this connection is the lapse-rate the decreasing temperature with height and the resulting small Richardson number (Richardson [15]) which is the criterion that turbulence in a gravitational field will increase. ${ }^{5}$ (In contrast, the high Richardson number in the stratosphere points to stable conditions.) The Richardson number is given as:

$$
\mathrm{R}_{i}=\frac{g\left(\frac{d T}{d z}+\Gamma g\right)}{\left(\frac{d v_{H}}{d z}\right)^{2} T}
$$

where $\Gamma$ is the adiabatic lapse rate $\left(\equiv \frac{1}{c_{p}}\right)$,

$c_{p}=$ specific heat at constant pressure,

$g=$ acceleration of gravity,

$T=$ temperature,

$v_{H}=$ horizontal velocity component of the wind, and $z$ is in the vertical direction.

As an example, the Richardson number for the lower troposphere, assuming the following characteristics:

$$
\begin{aligned}
c_{p} & =1.003 \mathrm{j} / \mathrm{g}^{\circ} \mathrm{K}, \\
T & =270^{\circ} \mathrm{K}, \\
\frac{d T}{d z} & =-5 \times 10^{-70} \mathrm{~K} / \mathrm{cm} \\
\frac{d v_{H}}{d z} & =1.9 \times 10^{-2} / \mathrm{sec},
\end{aligned}
$$

is

$$
\mathrm{R}_{i}(\text { troposphere })=0.98 .
$$

The only other region in the atmosphere where a decreasing temperature with height is found is in the mesodecline, at the top of which the airglow phenomenon is observed. Data are not so easily available to compute the Richardson number there, but the vertical change in the wind velocity is of the same order of magnitude as in the troposphere and instability would be expected. ${ }^{5}$ In other words, convection is likely to occur and it should reach roughly to the region of minimum temperature, the mesopause, and this is roughly where we find the green airglow.

If the emission changes can be correlated with the convective motions, then, on this picture, the airglow cells are to be considered as the top part of the convection elements in the mesodecline. It is $a$ priori not possible to predict in any detail how the penetration of air masses into the atomic oxygen concentration peak will affect the green emission, and only the conjecture is made here that this is the reason for the time variation of the airglow intensities.

It is generally agreed (Godske, Bergeron, Bjerknes, and Bundgaard [16]) that a Richardson number somewhat less than unity points to instability. But the exact critical value of the Richardson number is not known. 
Now, the mesopause is a rather shallow minimum. One even thinks of it as an isothermal region situated at around $85 \mathrm{~km}$. This is below the airglow height and this looks, at first sight, somewhat inconsistent. Two points should, however, here be taken into consideration. First, as Nicolet [10] points out, the height of the mesopause is not known to the nearest kilometer. Nicolet gives as the best estimate $85 \pm 10 \mathrm{~km}$. Second, if the region around 90 $\mathrm{km}$ is nearly isothermal, one would expect the convection elements to penetrate up through this layer, similarly as one observes eddies in the region just above the tropopause.

The interpretation of our data as wind implies that we have a component of ordered motion, a sort of "jet-stream," near the mesopause. On the other hand, this motion is certainly superposed on turbulence. The Reynold's number is

$$
\mathrm{R}_{e}=\frac{L \bar{v}}{\nu}
$$

where $L$ is a characteristic length, $\bar{v}$ is a characteristic velocity, and $\nu$ is the kinematic viscosity. With $\bar{v}=10^{4} \mathrm{~cm} / \mathrm{sec}$ and $\nu=4 \times 10^{4} \mathrm{~cm}^{2} / \mathrm{sec}$, a Reynold's number $\mathrm{R}_{\mathrm{e}}=0.25 L(L$ in $\mathrm{cm})$ is found. It is not obvious which characteristic length to put in, but if the boundary layers of the cells involved in the turbulence have a thickness as small as $1 \mathrm{~km}$, $R_{\mathrm{e}}=2.5 \times 10^{4}$ is found. Convection will tend to inhibit the turbulence, but since this is a region to which the convection only just penetrates, the characteristics of turbulence should probably manifest themselves. Evidence of this may be the rapid changes in luminosity often observed in the airglow cells.

On this picture, the maximum airglow intensity during a night may now come at any time, depending on the penetration of the instabilities into the maximum layer of the atomic oxygen distribution. Since the intensity will vary as $[n(0)]^{3}$ rather great variations may be expected, and due to the nature of turbulence relatively erratic time variations are not excluded. The green airglow is hence to be considered as a manifestation of "the weather in the mesodecline."

Obviously, too little is known about the dynamics of the regions involved to draw any definite conclusions, but this approach may be worth further study as more data on this part of the atmosphere become a vailable.

Attention is called to a study of 5577 by Tohmatsu [17] in which the Chapman reaction is assumed and the localized variations in atomic oxygen concentration are invoked to account for the observed patchiness of the night airglow, as well as the large scale temporal and geographical charges.

\section{Environmental Effects}

The fact that the 5577 airglow emission occurs in the ionosphere will now be considered. The electron density is small compared to the neutral gas density, but even in such a slightly ionized plasma new effects are likely to occur. This is the more probable since the plasma is pervaded by and moves in the earth's magnetic field.

The ionosphere has a general similarity to a gas discharge tube, and it is reasonable to inquire whether the conditions that lead to discharges in the laboratory are present in the ionosphere and will lead to luminous discharges that can be associated with airglow. ${ }^{6}$ It is characteristic of gas tube discharges that they can be explained only on the assumption that the electrons present are able to absorb energy selectively from an electric field between elastic collisions with heavy particles in the plasma. The essential feature is hence the presence of an electric field capable of accelerating electrons until they have enough energy to excite oxygen atoms. This leads to conditions in the plasma radically different from equilibrium and characterized by an electron gas temperature greatly in excess of the heavy particle temperature.

Several attempts have been made to formulate the problem. Chamberlain [18] postulated the existence of an electric field and determined the energy distribution curves and drift velocities of the electrons. He was able to show that auroral rays could be explained on such a discharge theory. Alfvén [19] and Wulf $[20,21]$ also discussed the mechanism by which the electric field could be set up. The problem includes two main facets: (a) The existence of an electric field capable of accelerating electrons, and (b) the heating of the electron gas and the excitation of the oxygen atoms.

\section{(a) Production of an electric field.}

Since it is implied here that the dynamics of the $100-\mathrm{km}$ region are of prime importance to an understanding of the green emission, those attempts to establish an electric field which involve extraterrestrial streams (see Alfvén [19]) will not be considered. This stage of the discussion is more in line with Wulf [21], inasmuch as the velocity $\mathbf{V}_{c}$ of the airglow cells as they move across lines of force of the earth's magnetic field $\mathbf{B}$ will be used. In doing so, an electric field is induced

$$
\mathbf{E}_{\text {ind }}=\mathbf{V}_{c} \times \mathbf{B}(\mathrm{emu})
$$

This field will tend to produce an electrostatic separation of charges until the polarization field $\mathbf{E}_{\text {pol }}$ thus created exactly balances $\mathbf{E}_{\text {ind }}$. Hence, in the frame of reference of the cell, there is no net electric field. Or, expressed in another way, the polarization field gives the charged particles (irrespective of sign) a drift velocity $\mathbf{V}_{d}$ in the direction of the motion $\mathbf{V}_{c}$, such that $\dot{\mathbf{V}}_{d}=\mathbf{V}_{c}$. The plasma therefore moves "downstream" as an entity, and

$$
\mathbf{V}_{d}=-\frac{\mathbf{E} \times \mathbf{B}}{B^{2}}
$$

6 It is a pleasure to acknowledge the many stimulating discussions on this topic with Dr. R. N. Thomas who first drew our attention to this similarity. 
In the absence of collisions, the electrons do not see any field and a heating of the electron gas is not possible. When the collisions are taken into account, the problem becomes quite difficult, and it is not at all obvious that the electrons can draw energy from the field. Wulf [21] avoided this basic difficulty by letting the circuit be closed through the $\mathrm{F}_{2}$ layer where the discharge took place. ${ }^{7}$ This would be physically understandable, but it is known the airglow emission comes where the wind speed $\mathbf{V}_{c}$ is measured, and this rules out the original Wulf approach. It may be recalled that Wulf used eq (10) and found that with a value of 0.5 gauss for the earth's magnetic field and a wind speed of $50 \mathrm{~m} / \mathrm{sec}$ a gradient of

$$
\begin{gathered}
\boldsymbol{E}=0.5 \times 5 \times 10^{3}=2.5 \times 10^{3} \mathrm{emu} \\
\text { or } \boldsymbol{E} \approx 10^{-5} \text { volts } / \mathrm{cm}
\end{gathered}
$$

would be created. As mentioned, this potential drop is not available for electron acceleration in this case, since an external path over which to discharge is needed.

Another possibility remains. Roach et al. [5] found evidence of a rotational velocity of the order of $400 \mathrm{~m} / \mathrm{sec}$ in the airglow cells. If this can lead to polarization effects in the cells, stronger fields (perhaps $10^{-4}$ to $10^{-3} \mathrm{v} / \mathrm{cm}$ ) might be expected to be set up between adjacent cells due to the great differential peripheral speeds. But the whole question is for the time being wide open, and no conclusion should be drawn before this problem has been thoroughly analyzed. The best for the moment is to assume the existence of the field and see what the results might be.

\section{(b) Acceleration of electrons and excitation of oxygen.}

If the same point of view as Chamberlain's [18] is taken, the question may be asked how a postulated electric field will affect the electron energy distribution. According to Chamberlain a gradient of order of magnitude between $10^{-5}$ and $10^{-4} \mathrm{~V} / \mathrm{cm}$ is sufficient to account for the acceleration of electrons to an energy capable of exciting the oxygen atoms in auroral rays. Now, it should be remembered that this conclusion is reached on the basis of deriving the electron energy distribution by a simplified theory (Smit [22]). The complete treatment of the problem. from the appropriate Boltzmann equation remains to be tackled.

The intensity of the 5577 emission is, according to Chamberlain, given by

$$
Q(5577)=\frac{A_{32}}{A_{32}+A_{31}} n(\mathrm{OI}) n_{e} S_{13} \frac{H}{10^{6}}
$$

where collisional deactivation has been neglected, and $Q$ is in rayleighs.

\footnotetext{
? At that time, it was believed that the green airglow originated at a height of approximately $200 \mathrm{~km}$.
}

Using a thickness of the emission layer equal to a scale height $(H=6.5 \mathrm{~km})$, the values given in the right side of figure $2\left(n_{e}=10^{2}\right.$ to $\left.10^{5}\right)$ are obtained; and with $H=100 \mathrm{~m}$ (to compare with Chamberlain's auroral conditions) the values in the left side of figure 2 (for $n_{e}=10^{4}$ to $\left.10^{8}\right)$. An energy $\epsilon$ ( $\equiv E \lambda$ where $\lambda$ is the mean free path) of approximately $10^{-1} \mathrm{v}$ is necessary to explain the airglow intensities. The extremely sharp dependence of the emission on this parameter $(\epsilon)$ may have an important bearing on the intensity variations in time and space of the airglow pattern, since inhomogeneities in E are likely to occur.

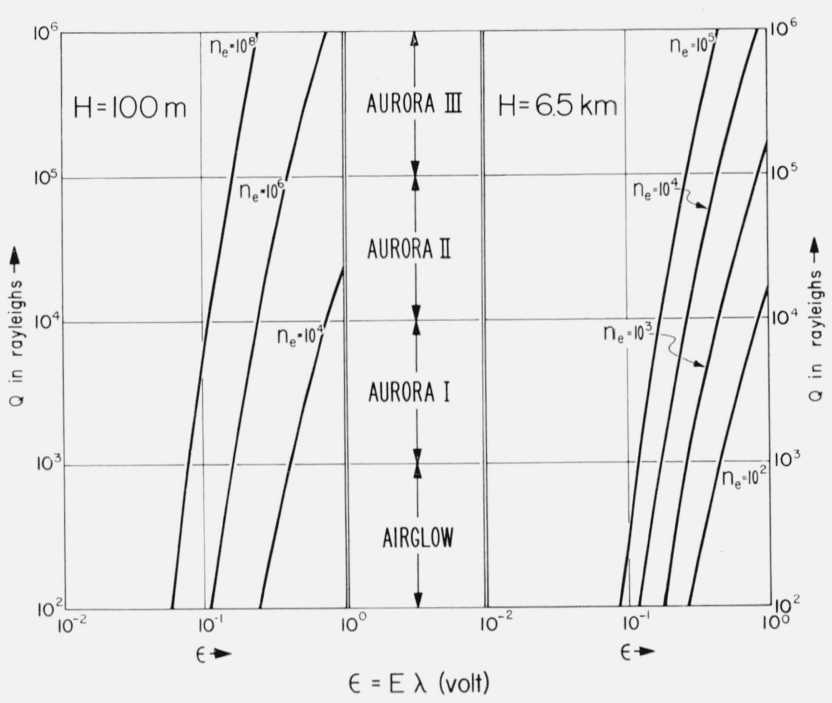

Figure 2. Predicted intensity of [OI] 5577 from eq (12).

The airglow is a normal feature of the "quiet" atmosphere in the $100-\mathrm{km}$ region, and it would here seem reasonable to compare it with other quietcondition phenomena of electromagnetic nature. The daily variations in the geomagnetic field are such a phenomenon. The variations can be explained in terms of a current system in the upper atmosphere, and rocket observations (Cahill [23]) show the existence of currents at $95-$ to $110-\mathrm{km}$ altitude. The question may therefore be asked whether electrons could be sufficiently accelerated in the electric field associated with this current system. On the night side of the earth where the airglow is observed, the current between equator and $40^{\circ}$ north is approximately 40,000 amp (Chapman and Bartels [24]). The width of the current sheet is hence $40 \times 110$ $\mathrm{km} \approx 4,000 \mathrm{~km}$. The thickness $l$ of the sheet is not too well known so the current density $j$ is written as

$$
j=\frac{I}{A}
$$


where the area $A=l \times 4 \times 10^{8} \mathrm{~cm}^{2}$ ( $l$ in $\left.\mathrm{cm}\right)$. Using Ohm's law,

$$
j=\sigma \mathbf{E}
$$

the corresponding electric field $\mathbf{E}$ is found provided the anisotropic electrical conductivity $\sigma$ is known. On magnetically disturbed days additional intense current systems at high latitudes can be estimated Matsushita [25]). Under quiet conditions (Matsuchita [26]) the electric field is estimated to be approximately $3 \times 10^{3} \quad \mathrm{emu}=3 \times 10^{-5} \mathrm{v} / \mathrm{cm}$. This is on the low side of what figure 2 indicates is necessary to produce the observed emission. On the other hand, the value $3 \times 10^{-5} \mathrm{v} / \mathrm{cm}$ is clearly a lower limit, since it is deduced using quiet geomagnetic conditions. The existence of magnetic disturbances means a greater electric field, and on the discharge theory this may be sufficient for the necessary heating of the electron gas.

It is hardly necessary to stress that this per se is no theory for the airglow, but the calculations indicate that it may be worth looking for an explanation of the $\lambda 5577$ emission along these lines.

\section{Summary and Conclusions}

The preceding paragraphs have examined existing theories and hypotheses of the green oxygen emission and presented promising alternatives. The sparsity of data available for some of the coefficients and parameters involved makes numerical calculations difficult, but some general conclusions can be drawn.

It is believed that "a modified photochemical theory" is capable of explaining the observed 5577 emission. The complex dynamics of the 90- to 100$\mathrm{km}$ region are not well known, but from what can reasonably be deduced, the "weather conditions" in the mesodecline are compatible with the observed variations of the 5577 emission in space and time.

Similarly, it is possible that the emission can be understood on the basis of "the environmental approach." In particular, the steepness of the curves in figure 2 indicates that small changes in electron density and/or the electric field (which probably occur in the low $E$ layer at night) will lead to rapid changes in brightness, and may explain space and time variations of the emission.

From the present study, one mechanism cannot be pointed to as the only possibility. This is perhaps not a very happy situation, but it may be that Nature is using two different mechanisms to generate the observed emissions.

The research reported in this paper has in part been sponsored by the Geophysies Research Directorate of the Air Force Cambridge Research Center, Air Research and Development Command, under Contract AF 19(604)-2140 to the High Altitude Observatory.

\section{References}

[1] J. P. Heppner, J. D. Stolarik, and L. H. Meredith, Rocket measurements at WSPG, New Mexico, July 5, 1956, Trans. Am. Geophys. Union 38, 394 (1957).

[2] F. E. Roach, L. R. Megill, M. H. Rees, and E. Marovich, The height of nightglow 5577, J. Atmospheric and Terrest. Phys. 12, 171 (1958).

[3] R. Tousey, Rocket Measurements of the night airglow, Ann. géophys. 14, 186 (1958).

[4] F. E. Roach, E. Tandberg-Hanssen, and L. R. Megill, The characteristic size of airglow cells, J. Atmospheric and Terrest. Phys. 13, 113 (1958).

[5] F. E. Roach, E. Tandberg-Hanssen, and L. R. Megill, Movements of airglow cells, J. Atmospheric and Terrest. Phys. 13, 122 (1958).

[6] M. J. Seaton, Theories of the airglow spectrum, Ann. geophys. 11, 232 (1955).

[7] S. Chapman, Some phenomena of the upper atmosphere, Proc. Roy. Soc. (London) A 132, 353 (1931).

[8] D. R. Bates, The physics of the upper atmosphere, The Earth as a Planet, ed. G. P. Kuiper, ch. 12 (University of Chicago Press, 1954).

[9] D. R. Bates and H. S. W. Massey, The basic reactions in the upper atmosphere I, Proc. Roy. Soc. (London) A 18\%, 261, (1946).

[10] M. Nicolet, La thermosphere, Ann. geophys. 15, 1 (1959).

[11] R. Garstang, Energy levels and transition probabilities in $p^{2}$ and $p^{4}$ configurations, Monthly Notices Roy. Astron. Soc. 111, 115 (1951).

[12] R. A. Minzner, W. S. Ripley, and T. P. Condron, U. S. extension to the ICAO standard atmosphere (1958).

[13] M. J. Seaton, Excitation processes in the aurora and airglow, J. Atmospheric and Terrest. Phys. 4, 295 (1954).

[14] R. G. Fleagle, Inferences concerning the dynamics of the mesosphere, J. Geophys. Research 63, 137 (1958).

[15] L. F. Richardson, The supply of energy from and to atmospheric eddies, Proc. Roy. Soc. (London) A 9y, 354 (1920).

[16] C. L. Godske, T. Bergeron, J. Bjerknes, and R. C. Bundgaard, Dynamic meteorological and weather forecasting, p. 459, (Am. Meteorol. Soc., Boston, Mass., 1957).

[17] T. Tohmatsu, The dynamical effect on the intensity of nightglow, Rept. Ionosphere Research Japan 12, 253 (1958).

[18] J. W. Chamberlain, Discharge theory of auroral rays, The airglow and the aurorae, ed. E. B. Armstrong and A. Dalgarno (Pergamon Press, 1955).

[19] H. Alfven, Cosmical electrodynamics (Oxford, Clarendon Press, 1950.)

[20] O. R. Wulf, On the relation between geomagnetism and the circulatory motions of the air in the atmosphere, Terrestrial Magnetism and Atmospheric Elec. 50, 185 (1945).

[21] O. R. Wulf, On the production of glow discharges in the ionosphere by winds, J. Geophys. Research 58, 531 (1953).

[22] J. A. Smit, Berechnung der geschwindigkeits-verteilung der elektronen bei gasentladungen in helium, Physica 3, 543 (1936)

[23] L. J. Cahill, Jr., Magnetic exploration of the upper atmosphere, Thesis, State University of Iowa (1959).

[24] S. Chapman and J. Bartels, Geomagnetism, Vol. I, Geomagnetic and related phenomena (Oxford University Press, 1951).

[25] S. Matsushita, Ionospheric variations associated with geomagnetic disturbances, J. Geomag. Geoelectr. 5, 109 (1953).

[26] S. Matsushita, On possible rocket observations of electric fields, Solar Research Memo No. 125, High Altitude Observatory, Univ. of Colorado (1959).

Boulder, Colo.

(Paper 63D3--29) 